\title{
Acheminement vers la parole unique
}

Autour du débat sur les langues en danger

On the Way of the Unique Language. On the Language Endangerment Debate

\section{Paolo Israel}

\section{(2) OpenEdition}

\section{Journals}

Édition électronique

URL : http://journals.openedition.org/etudesafricaines/123

DOI : $10.4000 /$ etudesafricaines. 123

ISSN : 1777-5353

Éditeur

Éditions de l'EHESS

Édition imprimée

Date de publication : 1 janvier 2001

Pagination : 815-832

ISBN : 978-2-7132-1394-6

ISSN : 0008-0055

Référence électronique

Paolo Israel, « Acheminement vers la parole unique », Cahiers d'études africaines [En ligne], 163-164 | 2001, mis en ligne le 22 novembre 2013, consulté le 05 février 2021. URL : http://

journals.openedition.org/etudesafricaines/123 ; DOI : https://doi.org/10.4000/etudesafricaines.123

(c) Cahiers d'Études africaines 


\section{Paolo Israel}

\section{Acheminement vers la parole unique}

\section{Autour du débat sur les langues en danger*}

\section{Le pouvoir des chiffres}

Les chiffres, il faut le reconnaître, sont bel et bien effrayants. D'ailleurs, ils sont l'entrée que tout ouvrage concernant la mort des langues nous offre, bien garnie avec des prospectus d'homologation de la diversité linguistique, voire culturelle, de l'œkoumène. Les chiffres, ainsi présentés, quoique souvent discordants dans les détails, vont tous dans le même sens. À la fin du $\mathrm{XXI}^{\mathrm{e}}$ siècle, la moitié des 5000 ou 6000 langues du monde pourrait ne plus être parlée, et une bonne partie d'entre elles pourrait être oubliée sans que leur mémoire en soit gardée. En outre, des 2000 ou 3000 langues survivantes, seules quelques centaines vont résister au cours du XXII ${ }^{\mathrm{e}}$ siècle (Hale 1998). C. Hagège (2000: 9) ouvre son livre en demandant (bien en gras) au lecteur s'il sait « qu'il meurt environ 25 langues chaque année. La moitié de la population mondiale parle l'une des vingt langues les plus parlées; $96 \%$ des langues du monde ne sont parlés que par $4 \%$ de ses habitants ${ }^{1}$; 50 langues sont parlées par une seule personne, et 4000 par moins de 20000 locuteurs (Crystal 2000 : 14). Tout cela fait soupçonner qu'un jour une seule langue (faut-il en douter? l'anglais, évidemment) dominera la planète (Dixon 1997: 140 ; Crystal 2000 : VIII).

Le premier but de la littérature abondante sur la mort des langues est d'avertir le lecteur à propos d'un phénomène à ne pas sous-évaluer. L'histoire, on le sait, est un cimetière de langues : le latin, l'assyrien, l'araméen

* À propos de Matthias Brenzinger, Endangered Languages in Africa, Koln, Rudiger Koppe Verlag, 1998 ; David Crystal, Language Death, Cambridge, Cambridge University Press, 2000 ; Robert M. W. DixON, The Rise and Fall of Languages, Cambridge, Cambridge University Press, 1997 ; A. Lenore Grenoble \& Lindsay J. WhaLeY, eds, Endangered Languages : Language Loss and Community Response, Cambridge, Cambridge University Press, 1998 ; Claude HagÈGE, Halte à la mort des langues, Paris, Odile Jacob, 2000 ; Daniel NetTle \& Suzanne Romaine, Vanishing Voices, Oxford, Oxford University Press, 2000.

Je remercie Jean-Loup Amselle pour ses conseils, et la lecture de la première version de cet article, et Francis Zimmermann pour son commentaire de l'article de M. SILVERSTEIN (1998).

1. Ce chiffre diminue jusqu'à $60 \%$, selon Nettle \& Romaine (2000). 
témoignent des destins fluctuants des civilisations et de leurs paroles. Les langues celtes, qui jadis dominaient l'Europe, luttent aujourd'hui pour être au moins reconnues par des bureaucraties parlant des langues d'origine latine. Toutefois, tous les chiffres sont là pour montrer que l'expansion de l'Europe sur les continents, à partir $\mathrm{du} \mathrm{Xv}^{\mathrm{e}}$ siècle, a déclenché une transformation du paysage linguistique de la planète qui n'a rien à voir avec ce qui s'est passé auparavant. C'est un phénomène massif et sans précédent qu'on nous présente, lié à la fragmentation du monde en États-nations de facto monolingues. À la suite des processus irréversibles de modernisation, de transformation de la nature et de mondialisation, il semblerait que le futur nous garde en sorte une version spéculaire de Babel où le mythe se renverse, et l'homme se punit lui-même de son orgueil en se mutilant du savoir local éparpillé sur la planète, savoir local que les innombrables peuples indigènes ont bâti au cours des millénaires. Les langues qui disparaissent, les voix qui se taisent à jamais sont des trésors immémoriaux de l'histoire de l'humanité qui sombrent dans l'oubli. Le déclin de l'Occident est déclenché par la destruction de son Autre : le châtiment destiné au ravageur est de sombrer dans le bouillon homologué que sa violence a généré.

En général, les chiffres sont loin d'être ingénus. Pour qu'on puisse compter, on doit pouvoir disposer d'entités discrètes, constituées par le biais d'une opération de découpage préalable. La mise en œuvre de cette opération d'individuation présuppose une prise de position ontologique explicite ou implicite sur la nature de l'objet même à isoler. L'histoire et l'idéologie du processus global de découpage et d'identification de la variété des langues du monde se résument dans le concept de grammatisation proposé par S. Auroux (1994). Puisque c'est par des chiffres que les paladins de la diversité linguistique de la planète défendent l'urgence et le sens de leur appel, puisque c'est par un comptage des destins d'entités discrètes réelles ou supposées (construites, identifiées, ou pas encore...) que s'articulent leur discours - le lien avec l'idéologie de la grammatisation, et avec son vecteur, l'écriture, est crucial pour la construction d'une telle rhétorique de défense. Par la mise en alphabet, la mise en grammaire et la mise en dictionnaire des langues passent soit l'évaluation générale de l'état des langues mondiales, soit le sort de chacune d'entre elles. Toutefois, dans l'idéologie linguistique des défenseurs des langues en danger, l'écriture joue un rôle aussi important qu'ambigu.

\section{Partages, sauvages, ravages}

Si le développement d'une littérature sur les langues en danger est un fait relativement récent, la peur liée à la modernité et à l'homologation culturelle, elle, n'est pas tout à fait nouvelle. Inspirée par la reviviscence de l'anthropologie de sauvetage opérée par Lévi-Strauss, sous l'égide de la thématique de l'ethnocide et du mythe du bon sauvage version Nambikwara 
(Amselle 1979), ainsi que par la renaissance de l'oralité des années 1960 et 1970 - en 1983 P. Zumthor, médiéviste de renommée, écrit un livre qui anticipe sur la production récente concernant la mort des langues. L'idéologie qu'il défend toutefois est symétrique et inversée par rapport à celle-ci. L'Introduction à la poésie orale se propose comme un traité sur la poétique de l'oralité et, pourtant, souvent des ambitions métaphysiques mettent en déroute l'approche phénoménologique qui voudrait animer le livre - l'oralité n'est plus alors une forme de l'investissement du corps dans l'activité langagière, mais un symbole de l'origine (selon l'étymologie latine de $o s$, oris, à la fois bouche et origine), et par là, de l'originaire, de l'authentique selon une suggestion d'origine psychanalytique ${ }^{2}$ : "Par la voix, nous restons de la race antique et puissante des Nomades » (Zumthor 1983: 285).

Si le Grand Partage entre oralité et écriture a été creusé pour fournir une explication de la domination de l'Occident sur le monde, et pour pouvoir nommer les «primitifs »d'une façon plus adaptée à l'époque, il ne fût pas difficile de renverser l'échelle des valeurs ${ }^{3}$. L'oralité - communautaire, pulsionnelle, directe — devint l'antithèse de la décadence de l'Occident scriptural et autistique (Ong 1982), par un parcours ambigu qui remonte à Platon, lequel critiquait l'écriture tout en l'utilisant pour faire sortir le monde grec de la phase semi-lettrée homérique 4 . Mais l'écriture est toujours aux aguets et l'oralité en danger.

«Depuis le XVII siècle, l'Europe s'est répandue sur le monde comme un cancer: subrepticement d'abord, mais voilà beau temps qu'il galope, ravage aujourd'hui dément de formes de vie, animaux, plantes, paysages, langues. À chaque jour qui passe, plusieurs langues au monde disparaissent : reniées, étouffées, mortes avec le dernier vieillard, voix vierges d'écriture, pure mémoire sans défense, fenêtres jadis grandes ouvertes sur le réel » (Zumthor 1983 : 282).

L'écriture, symbole de l'Occident, de même que la voix l'est des Autres, joue le rôle de ravageur. La production d'une numérologie apocalyptique ne manque pas chez Zumthor : "Cinq mille cultures anéanties, merveilleuse floraison d'humanité aujourd'hui fanée, profanée, radiée de nos cartes » (ibid.). L'écriture est la technique aliénée (et aliénante) qui tache la pureté de l'oralité. Les paradoxes de ce point de vue sont innombrables et ils n'échappent pas tous à Zumthor, qui choisit de vivre avec ${ }^{5}$. Et, toutefois, le paradoxe crucial porte sur la «nature graphique» de la connaissance anthropologique et linguistique des «peuples oraux » : c'est avec du papier

2. Zumthor rejoint les idées du linguiste «psychanalyste» I. FonAGY (1983).

3. L'oralité, conçue comme «seul passage de la bouche à l'oreille, jadis infériorisé, aujourd'hui valorisé-psychanalysé par certains comme la pulsion libératrice, qui reste dans le dualisme comme le blasphème reste dans la religion » (MESCHONNIC 1989: 246).

4. Dans le fameux final de Phedre. L'idée, selon laquelle Platon se trompait en critiquant l'écriture est de E. HAVELOCK (1986).

5. «Il peut sembler dérisoire d'écrire un livre sur la voix » est la première phrase que ZumTHOR écrit (1983: 9). 
et du crayon qu'on trace les cartes et qu'on compte les cultures. Il s'agit là d'un avatar de l'idée hegelienne selon laquelle la connaissance nie l'altérité de l'objet, l'absorbe et détruit l'objet en tant que tel. Si Hegel considérait ce passage comme inévitable, Zumthor se plaint de ses conséquences, tout en ne refusant pas ses présupposés. R. Rosaldo dirait que Zumthor est affecté par une sorte de «nostalgie impérialiste », soit le désir d'un passé qu'on a contribué à détruire (Rosaldo 1989 : chap. III) : bien qu'il n'ait rien détruit personnellement, en tant qu'écrivain il devrait se tenir responsable... Le cri de rage contre l'écriture est ambigu, puisque c'est grâce à elle que Zumthor est en mesure de dénoncer l'épanouissement des voix indigènes et qu'il arrive à repérer la renaissance de l'oralité dans les nouveaux médias, qui sont un sous-produit de la technologie de l'écriture. Notre destin dépend selon Zumthor de la capacité de bricoler les débris qu'ont laissés sur la planète «ces quelques siècles d'écriture ».

Selon H. Meschonnic (1982 : 284-285), c'est une définition sociologique de l'oralité, l' « identification de fait [...] entre oral et populaire » qui conduit Zumthor à présenter "l'inventaire de l'oralité comme inventaire d'un déclin ». Si on conçoit l'oralité comme « un primat du rythme et de la prosodie, avec sa sémantique propre, organisation subjective et culturelle d'un discours » (ibid. : 236), et pas comme le négatif de l'écrit, ou par le biais d'une définition phonique ou sociale (oral est le sonore, ce qui est transmis dans le circuit « oral-aural»), on peut échapper à cette contradiction et voir que l'oralité se réalise dans l'écrit et dans le parlé, modes du langage. L'oralité, en tant que présence du corps et du sujet dans le langage, est autant susceptible d'être en danger que l'est le langage lui-même.

\section{Les nouveaux paladins de Babel}

C'est vers le début des années 1980 que le monde officiel de la linguistique commence à se rendre compte de la portée et de l'urgence du phénomène de la mort des langues. Les premières études de cas importantes sont celles de N. Dorian (1981) sur les langues gaéliques d'Écosse, et de A. Schmidt (1985) sur la langue dyirbal ${ }^{6}$, qui restent, avec l'œuvre de H.-J. Sasse (1991), les exemples les mieux documentés et les plus cités sur la mort d'une langue. En 1989, N. Dorian publie le premier volume qui complète des articles théoriques et des études de cas relatifs à différentes zones géographiques, en faisant le point sur la question. C'est le début d'une tendance : dans les années 1990, sont publiés plusieurs volumes de ce genre (aux titres plutôt monotones, variations sur le thème de «la mort » et «le danger») (Aa. Vv. 1993 ; Robins \& Ulhenbeck 1991; Brenzinger 1992, 1998 ; Grenoble \& Whaley 1998 ; Bobaljick et al. 1996 ; Matsumura 1998).

6. Qui avait été précédemment décrite par R. DixON (1972). 
Les deux plus récents, publiés par Grenoble \& Whaley (1998) portant principalement sur les réponses des communautés au phénomène de la mort des langues et aux tentatives de préservation, et par Brenzinger (1998), recueil des interventions au symposium sur les langues en danger en Afrique, tenu au deuxième congrès de linguistique africaine de Leipzig $^{7}$, rentrent dans cette catégorie. Un débat dans la revue Language (Hale 1992 ; Ladefoged 1992 ; Krauss 1992 ; Dorian 1993), entre 1992 et 1993, donna une grande ampleur au sujet d'études dans le domaine de la linguistique académique. De la même façon, la revue La linguistique publia, en 1990, un numéro dédié aux langues en danger (Clairis 1990 ; Mounin 1992). Ce n'est que récemment que des ouvrages généraux, destinés à un public non spécialiste, paraissent, visant à mettre en vedette cette question et à sensibiliser les esprits. La publication de la première encyclopédie des langues en danger est prévue pour la fin 2001 (Moseley à paraître).

Bien que l'étude des langues en danger ne soit pas encore un sousdomaine institutionnalisé de la linguistique, avec ses centres et ses revues, il est tout à fait évident qu'elle est en train de conquérir un espace important dans le cadre académique de la discipline. Ce n'est pas un hasard si deux des principaux linguistes de France et d'Angleterre, Hagège et Crystal, ont publié des ouvrages de vulgarisation sur ce sujet presque au même moment. Par le biais de l'appel au sauvetage des langues moribondes, la linguistique occupe un espace médiatique qui est refusé aux théorisations post-chomskiennes ou aux recherches de terrain ${ }^{8}$. Seules les spéculations macro-génétiques de «l'école de Stanford » ont suscité un écho médiatique comparable (Ruhlen 1994 ; Nicolaï 2000 : 87-125). Les appels d'offre de l'UNESCO (avec le programme «Study of Endangered Langages »), du European Bureau of Lesser Used Languages et des nombreuses institutions privées (souvent religieuses), comme la Foundation for Endangered Languages de N. Ostler, Terralingua, ou le SIL International (anciennement Summer Institue of Linguistics) de Dallas (Crystal 2000 : 167-169), contribuent au développement du domaine. L'attention des institutions, des médias et des bailleurs de fonds pour le thème des langues en danger pourrait bien entraîner un basculement de l'axe majeur de la discipline - de la linguistique théorique vers la linguistique de terrain. Un plaidoyer pour ce basculement vient de certains secteurs de la linguistique de terrain (Dixon 1997 : 135-138 ; Brenzinger 1998 : 10), mais en général les nécessités de la linguistique théorique (la variété des données) sont l'un des éléments invoqués pour défendre l'urgence de la documentation sur les langues moribondes (Crystal 2000 : 55-65; Hale 1998).

7. M. BREnZINGER (1992) avait déjà publié un volume portant sur le cas de l'Afrique orientale.

8. On peut en tout cas se demander si la notoriété médiatique de Chomsky n'est pas davantage due à ses idées politiques qu'aux subtilités de son système théorique. 
Si le côté «dur» de la linguistique penche parfois vers l'informatique, l'intérêt pour les langues en danger marque une ouverture de certains secteurs de la linguistique (de terrain, mais pas forcément) aux autres sciences sociales, et en particulier à l'anthropologie, comme en témoigne l'effort conjoint de D. Nettle, anthropologue, et S. Romaine, linguiste (Nettle \& Romaine 2000). C'est dans l'anthropologie qu'on retrouve, en fait, une longue histoire de vocation militante axée sur le sauvetage des peuples menacés d'ethnocide, qui s'exprime aujourd'hui par un intérêt pour le rôle actif de l'anthropologue vis-à-vis des exigences politiques des communautés indigènes. "Advocacy» et «activism» semblent se proposer comme les nouveaux tenants du fieldwork anthropologique américain, bien que l'American Anthropological Association (AAA) ait décidé, lors de sa réunion de 1998, que la position d'activisme et de défense des communautés est un choix individuel de l'anthropologue et non une obligation éthique. Dans ce nouvel indigénisme, de même que, on le verra, dans la littérature sur les langues en danger' ${ }^{9}$, l'écologie joue un rôle important. Sur le versant médiatique, la série de films «Disappearing World » de la Granada Television ${ }^{10}$ est un bon exemple d'une variante de vulgarisation moderne de l'anthropologie de sauvetage, laquelle peut bien se marier avec les soucis écologistes de cette nouvelle linguistique.

Ce qui frappe le plus dans la lecture de cette abondante littérature est le décalage théorique et rhétorique entre les études de cas et les grandes généralisations des livres de vulgarisation. Les études de cas font honnêtement l'état de la situation de précarité dans laquelle se trouve une sousdiscipline née à la fin des années 1970, et se développant de façon intensive à partir des années 1990. En premier lieu, parce qu'il est difficile de définir les notions en jeu. Démêler les «langues » des «dialectes » est déjà une tâche qui pose d'innombrables difficultés théoriques et empiriques (peutêtre indépassables, faute de renoncer à ces concepts) — il s'agirait toutefois d'un stade indispensable pour faire l'état de la situation générale, du « language pool » de la planète (Crystal 2000 : 2-9). D'autre part, il est évident que le maintien «en bonne santé » d'une «langue indigène » dépend de questions plus générales relatives aux identités de groupe, à l'ethnicité et aux conflits de culture, notions qui continuent à poser des problèmes aux anthropologues qui les utilisent. Et, bien sûr, c'est tout le domaine des études créoles et du contact des langues qui est concerné par cette discussion.

Les «fondateurs » du domaine, Dorian et Schmidt, font très clairement l'état de la difficulté qu'ils ont à généraliser les données empiriques et les hypothèses théoriques relatives aux phénomènes de mort des langues. Dans l'introduction de Investigating Obsolescence, N. Dorian affirme que le domaine est encore très vague, que les mécanismes qui conduisent à la mort

9. La AAA a une section « anthropologie et environnement » qui s'intéresse aussi à l'indigénisme et à l'activisme.

10. Mais aussi la série presque homonyme «Vanishing Worlds» de Discovery Channel. 
des langues ne sont pas clairs, et qu'on n'est pas en mesure de prévoir la mort d'une langue, ni à partir de données contextuelles - le nombre de locuteurs ou la présence d'une situation de diglossie - ni à partir de données structurales, comme le niveau de simplification grammaticale d'une langue (Dorian 1989: 1-7). Parfois, une langue avec très peu de locuteurs peut survivre, et des transformations grammaticales très rapides, dans le sens d'une simplification, n'entraînent pas nécessairement la mort d'une langue. De même, A. Schmidt met en relief les nombreux paradoxes qui surgissent de l'étude de la langue dyirbal, et invite à une grande circonspection dans les conclusions générales (Schmidt 1985) ${ }^{11}$.

H.-J. Sasse (1992), dans une tentative de mise au point de la théorie de la mort des langues, met l'accent sur l'absence d'un cadre théorique : «As yet there is no theory of language death » (p. 7); «Extensive material on the process of language extinction, however, is available for only a limited number of European minority languages » (p. 8); «The entire field of linguistic obsolescence is still too poorly understood to allow for broader generalizations » (p. 24).

Pour ce qui est de l'Afrique, et en particulier de l'Afrique orientale, identifiée de façon quelque peu macabre comme «l'Eldorado des études des langues en danger », la situation semble aussi être très brouillée. Une évaluation de la situation africaine a été effectuée à partir d'une recherche bibliographique et d'un questionnaire envoyé à une centaine d'anthropologues et linguistes travaillant sur place, dont une trentaine ont répondu (Sommer 1992). "One of the immediate outcomes of the survey work was the observation that the available data on most of the minority languages taken into consideration are either scanty, inconsistent, or even contradictory. For many of them (languages) hardly more is known than the fact that they are threatened by extinction »(Brenzinger 1998: 123) ${ }^{12}$.

Par ces appels à la prudence, les auteurs en question ne veulent pas du tout nier que soit en cours actuellement un phénomène de mort massive des langues, ni le sous-évaluer, puisqu'ils l'ont choisi comme domaine d'études privilégié. Toutefois, ses formes, son évaluation présente et future, et ses dimensions effectives semblent difficiles à cerner, ce qui rend improbables et aventureuses les prévisions et les grandes fresques.

\section{Les faces du Prisme de Humboldt}

En revanche, dans les grands ouvrages, la verve propagandiste ne fait pas dans les nuances: elle procède fortement d'un mariage entre la rhétorique

11. "In terms of the type of change, there is not much difference between dying Dyirbal and other normal languages » (p. 212); «We should be cautious [...] because there is, as yet, insufficient empirical description of language death situations » (p. 215).

12. Et toutefois, M. Abdulaziz propose de limiter toutes les activités de recherche linguistique en Afrique à la documentation des langues en danger! (BRENZINGER 1998 : 9). 
des grands chiffres et un cadre théorique écolo-relativiste. Si l'œuvre de Zumthor se situait dans une optique toute moderne - la critique de la modernité à l'aide de ses propres termes —, la littérature sur les langues en danger basculerait entre une anthropologie de sauvetage de saveur boasienne et un contexte conceptuel post-moderne: l'indigénisme, le relativisme culturel et l'écriture étant ses points d'ancrage idéologiques.

W. von Humboldt a fourni une des plus efficaces expressions du relativisme linguistique (dans le sens de la relation de la vérité au langage) dans les termes de la philosophie moderne ${ }^{13}$. Les idées de Humboldt pénétrèrent dans le milieu de l'anthropologie linguistique (et de l'anthropologie tout court) américaine, par le biais de Boas, Sapir et Whorf — la mal nommée « hypothèse Sapir-Whorf » étant débitrice du concept philosophique de weltanschauung appliqué aux langues. Une métaphore humboldtienne saisit très efficacement une des argumentations principales des défenseurs des langues en danger : "Car toutes les langues ensemble ressemblent à un Prisme dont chaque face montrerait l'univers sous une couleur différemment nuancée » (Humboldt 1812: 321).

Si chaque langue porte sur le monde un regard idiosyncrasique et irréductible aux autres, la réduction des faces du Prisme est une perte quantitative de «points de vue» sur l'univers ${ }^{14}$. Chaque nuance est à préserver, faute de quoi on ne se retrouvera qu'avec quelques couleurs sur la palette pour peindre le monde. «Why should we care ? » est une des cinq questions autour desquelles est bâti le pamphlet de Crystal (2000: 68-91). Les réponses sont multiples : dans les langues se sédimente l'histoire (p. 40), les langues sont nécessaires aux grandes théories linguistiques (p. 57) ${ }^{15}$, les langues expriment l'identité (p. 36). Toutefois ce sont surtout « les fantômes de Whorf et Sapir» (p. 54) qui animent ces pages. Il faut défendre les langues parce qu'elles sont « an unprecedented event and a unique encapsulation of a world view» (p. 54). Le concept est répété chez C. Hagège (2000) avec une rhétorique plus vivace : «Les langues reflets de l'infini » (p. 17) ; «Structures cognitives [...] qui portent les marques des opérations par lesquelles s'exprime l'univers des choses sensibles et des concepts » (p. 15). Il en est de même chez Nettle \& Romaine (2000 : 25 et 29). K. Hale 1992), dans un article paru dans Language et publié dans le recueil de

13. Humboldt n'était pas tout à fait un relativiste au sens vulgaire du terme (ou au sens des sophistes), puisque son souci d'enraciner la vérité dans « chaque langue déterminée » (TRABANT 1999 : 39) s'accompagnait d'une recherche philosophique de nature transcendantale. En somme, on ne peut voir dans son travail très complexe et d'un grand intérêt, qu'une «préfiguration » de l' « hypothèse » de Sapir et Whorf.

14. Faut-il le préciser, Humboldt n'avait probablement rien de semblable à l'esprit quand il écrivait la phrase qu'on a citée.

15. Sur ce point, il y a un accord presque universel entre les commentateurs : faute d'une linguistique de sauvetage, pas de travail pour les linguistes théoriques non plus! 
Grenoble et Whaley (1998 : 192-216), défend le même principe de la grammaire comme dépositaire de visions du monde différentes.

Il n'est pas question de sous-estimer les drames linguistiques en cours dans d'innombrables endroits de la planète (qui ne nous semblent pas différents, en tout cas, de ceux qu'ont dû éprouver Astérix et Obélix une fois que leur village fût conquis par les Romains). Il s'agit de s'interroger sur les bases théoriques des livres de combat à prétentions généralisantes. On donne volontiers la parole, in extenso, à M. Silverstein qui, dans un récent article de synthèse, fournit une saisissante critique théorique des métaphores écologiques appliquées au langage :

«As a political position, it may be. But in terms of professional - that is, scientific - self-justification, for the applicability of the metaphor, on the other hand, we generally find merely an untheorized folk view. The position is generally rationalized by a most naïve Whorfianism about culture and so-called "world view" (itself an ideological term that bespeaks a lack of contact with serious anthropological, linguistic anthropological or philosophical discourse since probably 1950 !); these are somehow seen to be embodied in each particular language's denotational structure - of all things ! - of which "world view" must somehow be a function. This from the same folks who, as theoreticians, value the language-specific only in terms of a universalist and explicitly or implicitly formalist scientific project! » (Silverstein $1998: 422$ ).

Le nœud théorique de la question est donc une idéologie linguistique vieille de cinquante ans. Si une langue peut être conçue comme un «code dénotatif »(denotational code), soit un ensemble structuré composé par une grammaire et un dictionnaire, toutefois la «sémiose indéxicale » joue un rôle crucial (p. 407). Silverstein oppose les communautés de langue (language community) aux communautés de parole (speech communities), au sens ou les premières sont bâties sur l'affiliation à un code dénotatif et les deuxièmes sur des «perduring, presupposables regularities of discursive interaction » (ibid.), soit l'indexicalité. Ce concept clé de la linguistique et de l'anthropologie linguistique contemporaine indique de façon spécifique les références à la subjectivité dans le discours, par le biais des embrayeurs, du corps, du style. Le concept de rythme, proposé par H. Meschonnic ${ }^{16}$, quoique de nature tout à fait différente, exprime le même intérêt pour une ouverture de la structure au corps, au sujet, à l'expression.

Le point qu'il faut retenir est que, dans le langage, l'expression d'une subjectivité — et des «visions du monde» subjectives — ne passe pas forcément par la grammaire et le vocabulaire. Il y a plus que cela dans les langues, autour des langues, entre les langues. L'image d'un monde fragmenté dans un nombre bien défini d'entités discrètes — les 5000 ou 6000

16. «Je définis le rythme dans le langage comme l'organisation des marques par lesquelles les signifiants, linguistiques et extralinguistiques (dans le cas de la communication orale surtout) produisent une sémantique spécifique, distincte du sens lexical, et que j'appelle la signifiance, c'est-à-dire les valeurs propres à un discours et à un seul » (MeschONNIC 1982 : 217). 
langues - est un effet de l'idéologie de la grammatisation (Auroux 1994), soit de la réduction des langues du monde à des huis clos « dénotatifs » formés par une grammaire et un dictionnaire, calqués sur la structure de la langue (de la grammaire) latine, et fonctionne en parallèle à l'œuvre de « réduction à l'ethnie » des populations du monde mis en œuvre par l'ethnologie (Amselle 1990). C'est pour cela qu'on tend à refuser les conceptions « essentialistes » des langues, et on a des difficultés aussi à manier des concepts pourtant modernes et importants comme celui de code switching, qui présupposent qu'on a à faire avec des codes figés, des entités autonomes et séparées ${ }^{17}$.

Ce que les phantasmes whorfiens inspirent aux paladins des langues en danger est qu'il faut protéger la mosaïque actuelle des "codes dénotatifs », parce que dans chacun d'entre eux se cache un trésor de savoir. Ce qu'il y a autour de l'ensemble grammaire-dictionnaire est trop souvent oublié, ce qui empêche de percevoir la borne qui sépare transformation et mort. C'est un des paradoxes mis en évidence par A. Schmidt (1985: 3) : «On the one hand a no-longer-developing language is seen as a "dead" language ; and on the other hand a language which changes too rapidly (or, if over a long period, even gradually), is often no longer regarded as the "same language". »

Les remèdes proposés sont basés - au contraire de ce qui se passait chez Zumthor - sur la grammatisation (et donc l'écriture) : il faudra produire des grammaires et des dictionnaires pour chacune des langues du monde (en les comptant préalablement), pour les documenter et pour qu'ils fonctionnent comme un instrument de défense (ou, dans le cas extrême, de résurrection) d'une langue. Il faut surtout compter les faces du Prisme de Humboldt et faire en sorte qu'il ne bouge plus.

\section{Arbres, gènes, germes et autres figures biologiques}

À côté du whorfisme, le deuxième point d'ancrage des paladins des langues en danger est l'écologie, selon l'analogie entre langues et espèces. Par cette métaphore, les langues sont des organismes vivants qui, comme les espèces qui peuplent la planète, sont susceptibles de disparaître, sont en péril d'extinction. Le premier chapitre du volume de $\mathrm{C}$. Hagège reconstruit historiquement l'origine de ce parallélisme, origine qu'il fait remonter au vitalisme linguistique de A. Schleicher (pp. 23-37). Tout le volume, qui tourne autour de l'opposition entre vie et mort, a une saveur darwinienne : les langues, comme les espèces, sont en lutte pour la vie. Vanishing voices (Nettle \& Romaine 2000) est entièrement bâti sur une hypothèse écologique originale. Selon les deux auteurs, la biodiversité et la « glottodiversité » se redoublent sur la surface de la planète: les aires avec plus d'espèces vivantes sont

17. M. SiLVERSTEIN (1998: 411) parle de «ill-named "code-switching"». 
aussi celles où l'on parle plus de langues diverses (en gros, les aires comprises entre les deux tropiques, et en particulier la Nouvelle Guinée et l'Afrique équatoriale). Ce phénomène - que les auteurs nomment «diversité biolinguistique »- s'explique par le fait que les langues s'adaptent aux milieux naturels (ce qui est attesté par les recherches de l'ethnoscience sur les taxinomies naturelles), et que donc, dans les zones où il y a les écosystèmes plus différenciés, les langues tendent aussi à se différencier en s'adaptant à l'environnement (ce qui vaut, évidemment, surtout — ou seulement? - pour le lexique). Il est difficile, en premier lieu, de comprendre comment il est possible de penser à la fois qu'une langue oriente la vision du monde (le whorfisme naît du proto-idéalisme allemand), et que son existence indépendante soit le produit de l'articulation des écosystèmes locaux (ce qui revient à une sorte de matérialisme brutal). Quoi qu'il en soit, cette acrobatie intellectuelle conduit directement à l'exaltation de la figure de l'indigène ${ }^{18}$ (chasseur ou cueilleur) et de son lien profond avec la nature, lien qui s'exprime dans sa langue «ancestrale». S'agit-il encore une fois du «bon sauvage»? L'histoire « indigéniste » que Nettle et Romaine nous content est assez argumentée, et s'appuie sur le grand récit des dernières 13000 années récemment esquissé par J. Diamond (1997), sous l'égide théorique du réductionnisme écologique ${ }^{19}$. Ce récit qui s'appuie en grande partie sur la grande histoire génético-linguistique du monde, cristallisée dans le projet stanfordien de L. Cavalli-Sforza (1996), lui a valu un prix Pulitzer.

Diamond se propose ainsi d'expliquer la conquête européenne du monde à partir de grandes données écologiques comme la distribution des espèces animales, le climat, l'orientation géographique des continents ${ }^{20}$. La domestication des animaux favorise la proximité et donc la résistance aux maladies, une organisation sociale plus complexe, et donc des armes plus puissantes. Selon Nettle et Romaine, les deux vagues de colonisation qui ont parcouru la planète, la vague «biologique » (néolithique) et la «vague économique » (moderne), ont altéré l'équilibre linguistique des peuples aborigènes (chasseurs ou cueilleurs), la première de façon transitoire quoique profonde (l'impact des langues bantu sur les langues khoisan en Afrique, récemment), la deuxième de façon probablement irréversible (chap. 5, 6). Les transformations linguistiques actuelles sont ainsi en train de détruire un équilibre

18. « Native », of course. C'est un des problèmes de l'anthropologie américaine que d'avoir adopté ce concept qui n'est pas moins ambigu et potentiellement dangereux que bien d'autres refoulés de l'évolutionnisme, «sauvages » ou «primitifs » d'antan.

19. Qui, selon les souhaits de l'auteur, devrait conduire à la transformation de l'histoire en science «dure » - on en arrive presque à la psycho-histoire d'Asimov...

20. Il n'est pas question d'entrer dans le détail de ce travail important. On observe, toutefois, qu'en traitant des temps qui nous sont plus proches (et à une échelle temporelle plus réduite), Diamond est obligé de mettre en jeu de nombreuses causes «culturelles » (historiques) qui ne se laissent pas aisément reconduire à des données écologiques. 
millénaire et de disperser un patrimoine de connaissance linguistique et écologique accumulé au cours de l'histoire de l'humanité. La possibilité d'un développement soutenable passe, selon les auteurs, par le sauvetage du savoir écologique conservé par les langues ancestrales des peuples indigènes ${ }^{21}$.

La référence à J. Diamond, et donc, indirectement, à L. Cavalli-Sforza, débouche sur un cercle d'argumentations assez intéressant. Les hypothèses génétiques de Cavalli-Sforza sont corroborées par la coïncidence avec les macro-familles linguistiques de J. Greenberg et de son école (Cavalli-Sforza 1996 : chap. 5). Or, le plus brillant élève de Greenberg, M. Ruhlen, dans un ouvrage très peu académique ${ }^{22}$, défend l'idée qu'il est possible de retracer la langue-mère, laquelle aurait donné naissance à toutes les langues du monde. L'implication de cette idée est que les langues qu'on parle actuellement contiennent des traces de la langue-mère, et sont donc véritablement des trésors historiques permettant de faire remonter notre histoire jusqu'à la langue (africaine) d'Adam et d'Ève ${ }^{23}$. D'ailleurs, L. Cavalli-Sforza luimême se range parmi les pessimistes : «Beaucoup de populations (et des cultes et de langues) sont en train de s'éteindre à cause des migrations qui, aujourd'hui comme jamais, mêlent les gênes, les langues et les cultures [...]. La richesse humaine — biologique et culturelle — de ces populations à risque doit être sauvegardée avec tous les moyens » (Cavalli-Sforza 1997: XVIII).

Ce qui fait problème, c'est la notion d'arbre généalogique, indissolublement liée à celle d'évolution. L'évolution biologique et le changement linguistique se font sur des échelles historiques radicalement différentes, ce qui met en abyme l'idée d'un parallélisme entre espèce et langue. En outre, toutes les langues peuvent s'hybrider ${ }^{24}$ entre elles, tandis qu'entre un chat et un éléphant il n'y a pas de croisement possible. À la notion d'évolution arborescente est dédié un ouvrage récent du linguiste australien R. Dixon (1997) qui, tout en s'alignant sur les prévisions catastrophistes des écolinguistes, présente un modèle alternatif pour la compréhension des transformations linguistiques. Les critiques véhémentes de Dixon visent les théories macrogénétiques de l'école russe des «nostraticistes» et des greenbergiens ${ }^{25}$.

21. En réponse, I. Buruma (2001: 26) invoque l'exemple des Inuits : " Another expression of their identity is shooting rare Bowhead whales with 50 caliber hunting rifles. [...] The hunts are not just for the meat. They are defended on cultural grounds: shooting whales is deemed essential for the preservation of identity. »

22. Ce qui n'est pas nécessairement un défaut... Pour une analyse de la rhétorique de Ruhlen, voir Nicolaï (2000 : 31-35).

23. Ruhlen n'est apparemment pas gêné par le manque de documentation dénoncé par les linguistes de terrain travaillant sur les langues en danger, selon lesquels une bonne moitié des langues du monde n'est pas suffisamment documentée.

24. Ce mot trahit un esprit essentialiste (le mélange de deux entités différentes), mais il n'y a pas le choix, ce sont les termes du discours qui l'exigent.

25. Le «nostalgique» serait une macro-famille regroupant la plupart des langues eurasiatiques. DixON (1997 : 32-35) critique aussi la fameuse classification des langues africaines de Greenberg qui est l'un des arguments invoqués par Ruhlen 
Selon Dixon (1997: 29), le modèle arborescent d'évolution linguistique n'est applicable que sur des échelles temporelles limitées, faute de quoi on parlerait aujourd'hui quelques millions de langues différentes ${ }^{26}$. Dans le modèle qu'il propose («punctuated equilibrium model »), de brèves phases de «punctuation »- caractérisées par une évolution arborescente, coïncidant avec de grands phénomènes de transformations sociales —, alternent avec de très longues phases d'équilibre, caractérisées par la diffusion des traits linguistiques. La reconstruction des macro-familles et de la languemère est donc une illusion ou une imposture intellectuelle. Les langues du monde sont le produit de processus alternés de séparation et de brassage, et une langue est en mesure de générer la diversité en un laps de temps relativement bref. En dépit de sa théorie, Dixon croit que la phase de ponctuation actuelle pourrait conduire à la disparition de la diversité linguistique du monde.

\section{Nouvelles frontières de la grammatisation}

La rhétorique des langues en danger ${ }^{27}$ se présente et se définit comme une nouvelle vague radicale de grammatisation, destinée à s'effectuer à l'échelle globale, le plus rapidement possible. Il ne faut pas s'étonner que le web soit un de ses moyens privilégiés. Mais, en revanche, on peut s'étonner que la source privilégiée d'informations sur la situation linguistique du monde soit la revue Ethnologue ${ }^{28}$, publiée par le SIL International, institution américaine d'inspiration religieuse. L'inventaire des langues proposé par Ethnologue est surtout un recueil de noms; les critères principaux d'évaluations de l'état de santé d'une langue sont la présence d'un dictionnaire, d'une grammaire et de la traduction de la Bible. Soit son niveau de grammatisation. Ethnologue fait un travail méritoire et important, mais les critères de scientificité qu'il offre ne permettent pas de fonder une évaluation du destin des langues de la planète. Autre site, autre histoire. Le Rosetta project est la dernière entreprise de grammatisation et de conservation des langues du monde. Les organisateurs du projet sont en train de constituer un équivalent moderne de la pierre de Rosetta ${ }^{29}$ : un disque de nickel, garanti pour 2000 ans,

et Cavalli-Sforza pour défendre la validité de la classification des langues amérindiennes (selon l'idée que si on a d'abord refusé et ensuite accepté la classification africaine, on va faire de même avec l'amérindienne).

26. En prenant comme exemple l'indo-européen, ou le proto-australien, qui a généré un millier de langues en 5000 ans.

27. On entend par là le discours global sur l'homologation linguistique de la planète, et non pas le problème des transformations linguistiques radicales qui est une réalité dramatique dans de nombreux contextes locaux. "Deaths are always sad events. But I am not sure the ecolinguists always deplore these losses for the rights reasons » (BURUMA $2001: 24$ ).

28. http ://www.sil.org/ethnologue/

29. http ://www.rosettaproject.org 
sur lequel seront archivées, pour les générations futures, un millier de langues, par le biais de descriptions linguistiques, d'une traduction de la Genèse, de textes vernaculaires commentés, et d'une liste Swadesh ${ }^{30}$. L'apocalypse est proche.

Toutefois, il en est qui ne croient pas à l'apocalypse linguistique. Ou qui pensent que la tendance à la diversification culturelle et linguistique n'est et ne sera jamais en péril ${ }^{31}$. Le bilinguisme, qui est une caractéristique constante du paysage linguistique d'Afrique, commence à se diffuser dans une réalité supranationale comme la Communauté européenne. C'est peutêtre vers des politiques bilingues qu'il faut se diriger, tout en confiant, avec I. Buruma (2001) que «just as we cannot stop ourselves from rebuilding the tower of Babel, it will be knocked down again and again ${ }^{32}$.

EHESS, Paris.

\section{BIBLIOGRAPHIE}

$\mathrm{AA}, \mathrm{Vv}$.

1993 Les langues menacées, Actes du XV Congrès international des linguistes, Québec, Université Laval, 9-14 Août 1992, vol. I-IV, Sainte Foi, Presses de l’Université de Laval.

Amselle, J.-L., ed.

1979 Le sauvage à la mode, Paris, le Sycomore.

1990 Logiques métisses, Paris, Payot.

Auroux, S.

1994 La révolution technologique de la grammatisation, Liège, Mardaga.

Bobaljik, J., Pensalfini, R. \& Storto, L., eds

1996 Papers on Language Endangerment and the Maintenance of Linguistic Diversity, MIT Working Papers in Linguistics, vol. 28, Cambridge, MIT Press.

30. Il s'agit des listes de vocabulaire de base sur lesquelles se fondent la glottochronologie - qui est l'équivalent en linguistique du carbone14 - et les spéculations génétiques de Greenberg.

31. "They may not be based on ethnicity or language, but the differences remain. Societies will always produce subgroups as varied as computer nerds, valley girls, and drug pushers, who think and behave in different ways » (LADEFOGED 1992 : 810). Pour une approche critique, voir aussi MounIN (1992).

32. Il pourrait sembler paradoxal de conclure de façon optimiste un article sur les langues en danger avec une citation en langue anglaise. Il faut vivre avec, comme Zumthor (mais les paladins des langues en danger n'écrivent-ils pas en anglais?). 
BRENZINGER, M., ed.

1992 Language Death, Factual and Theoretical Explorations with Special Reference to East Africa, Berlin-New York, Mouton de Gruyter.

1998 Endangered Languages in Africa, Köln, Rudiger Koppe Verlag.

BURUMA, I.

2001 «The Road to Babel», New York Review of Books, XLVIII (9) : 23-26.

CAvalli-Sforza, L.

1996 Gènes, peuples et langues, Paris, Odile Jacob (Nouvelle préface: Storia e geografia dei geni umani, Milano, Adelphi, 1997).

Cavalli-Sforza, L., Menozzi, P. \& Piazza, A.

1994 The History and Geography of Human Genes, Princeton, Princeton University Press.

Clairis, C.

1990 «Le processus de disparition des langues », La Linguistique, 27 (2) : 3-13.

CRystal, D.

2000 Language Death, Cambridge, Cambridge University Press.

DiAMOND, J.

1997 Guns, Germs and Steel. The Fates of Human Societies, New York-London, Norton.

DixON, R.

1972 The Dyirbal Language of North Queensland, Cambridge, Cambridge University Press.

1997 The Rise and Fall of Languages, Cambridge, Cambridge University Press.

DORIAN, N.

1981 Language Death: The Life Cycle of a Scottish Gaelic Dialect, Philadelphia, The University of Pennsylvania Press.

1989 Investigating Obsolescence: Studies in Language Contraction and Death, Cambridge-London, Cambridge University Press.

1993 «A Response to Ladefoged's Other View of Endangered Languages », Language, 69 (1) : 575-579.

FONÁGY, I.

1983 La vive voix. Essais de psycho-phonétique, Paris, Payot.

Grenoble, L. \& Whaley, L., eds

1998 Endangered Languages : Language Loss and Community Response, Cambridge, Cambridge University Press.

Hagège, C.

2000 Halte à la mort des langues, Paris, Odile Jacob. 
HaLe, K.

1992 «Language Endangerment and the Human Value of Linguistic Diversity », Language, 68 (1) : 35-42.

1998 « On Endangered Languages and the Importance of Linguistic Diversity », in L. Grenoble \& L. Whaley, op. cit : 192-216.

Havelock, E. A.

1986 The Muse Learns to Write. Reflections on Orality and Literacy from Antiquity to the Present, New Haven-London, Yale University Press.

Humboldt, W., von

1812 «Essai sur les langues du Nouveau Continent», in A. Leitzmann et al., eds, Wilhelm von Humboldt Gesammelte Schriften, im Auftrag der Königlichen Preußischen Akademie der Wissenschaften, Berlin, Behr, vol. III : 300-342.

Krauss, M.

1992 « The World's Languages in Crisis », Language, 68 (1) : 4-10.

LADEFOGED, P.

1992 «Another View of Endangered Languages », Language, 68 (4) : 809-811.

Matsumura, K., ed.

1998 Studies in Endangered Languages, Tokyo, Hituzi Syobo.

Meschonnic, $\mathrm{H}$.

1982 Critique du rythme. Anthropologie historique du langage, Paris, Verdier.

1989 La rime et la vie, Paris, Verdier.

Moseley, C., ed.

à paraître Encyclopedia of the World's Endangered Languages, Surrey, Curzon Press.

Mounin, G.

1992 «Sur la mort des langues », La Linguistique, 28 (2) : 149-158.

Nettle, D. \& Romaine, S.

2000 Vanishing Voices: The Extinction of the World's Languages, Oxford, Oxford University Press.

NicolaÏ, R.

2000 La traversée de l'empirique, Paris, Ophrys.

ONG, W. J.

1982 Orality and Literacy. The technologizing of the World, London-New York, Methuen. 
Robins, R. H. \& Uhlenbeck, E. M., eds

1991 Endangered Languages, Oxford-New York, Berg.

RosALDO, R.

1989 Culture and Truth, Boston, Beacon Press.

RUHLEN, M.

1994 The Origin of Language. Tracing the Evolution of the Mother Tongue, New York, Wiley.

SASSE, H.-J.

1991 Arvanitika. Die albinisme Sprachreste in Griechenland, vol. 1, Wiesbaden, Harrasowitz Verlag.

1992 Theory of Language Death, in M. BRENZINGER, op. cit. : 7-30.

SCHMIDT, A.

1985 Young People's Dyirbal : an Example of Language Death from Australia, Cambridge-London, Cambridge University Press.

Silverstein, M.

1998 «Contemporary Transformations of Local Linguistic Communities », Annual Review of Anthropology, 27 : 401-426.

SOMMER, G.

1992 «A Survey of Language Death in Africa», in M. Brenzinger, op. cit. : 301-418.

Trabant, J.

1999 Traditions de Humboldt, Paris, Éditions de la Maison des sciences de l'homme.

ZuMTHOR, P.

1983 Introduction à la poésie orale, Paris, Éditions du Seuil.

\section{RÉSUMÉ}

Le phénomène de la mort des langues est en train de transformer le paysage linguistique de la planète. Toutefois, les grandes fresques qui prévoient l'homologation linguistique et culturelle à l'échelle mondiale sont le produit d'une idéologie linguistique qui n'est pas neutre. Cette idéologie s'articule sur la grammatisation au sens de S. Auroux, sur le relativisme linguistique, notamment dans sa version würmienne ; sur une métaphore écologiste entre langues et espèces vivantes en danger d'extinction et sur le modèle arborescent de l'évolution linguistique. Sans sous-évaluer les problèmes des transformations linguistiques, on essaie dans cet article d'analyser et de critiquer les présupposés théoriques des généralisations apocalyptiques. 


\section{ABSTRACT}

On the Way of the Unique Language. On the Language Endangerment Debate. - Language death is transforming the world's linguistic landscape. However, the generalizing works depicting a global linguistic and cultural homologation are the product of a biased linguistic ideology. This ideology is based on grammatization (in S. Auroux's terms), on whorfian linguistic relativism, on an ecological metaphor between endangered languages and endangered species and on the family tree model of linguistic evolution. This paper, while not underestimating language death, addresses and criticizes the theoretical foundations of these apocalyptic discourses.

Mots-clés/keywords: écolinguistique, écriture, grammatisation, hypothèse SapirWhorf, indexicalité, indigénisme, langues en danger, linguistique génétique, mort des langues, oralité/Ecolinguistics, literacy, grammatisation, Sapir-Whorf hypothesis, indexicality, indigenism, language endangerment, genetic linguistics, language death, orality. 